\title{
Exposure Dose Form
}

National Cancer Institute

\section{Source}

National Cancer Institute. Exposure Dose Form. NCI Thesaurus. Code C83111.

The dosage form of the exposure. 